it was found that the dummy medication intensified high-amplitude finger tremor and, as a result, tremorrate after a sedative was also greater than when no treatment was given.

It is obvious from these trials that difficulty may arise in defining the base-line for the estimation of pharmacodynamic actions in man, hence in making absolute measurements of drug action. In this study, because drug and placebo effects were opposed, drug action could be measured in terms of the extent by which the drug diminished the placebo reaction. In the situation in which drug effect is in the same direction, but exceeds placebo effects, the question of how to obtain an absolute measure of drug effect is a difficult one, for the results may not indicate whether the drug action supplements placebo effect or accompanies it. In other cases, in which the subtraction indicates no effect in excess of placebo effect, it does not rule out the possibility of the complete masking of drug effect. This also brings up the question of the sensitivity of the method requisite for drug evaluations since, as the relative proportions of placebo and pharmacodynamic actions in the total effect change, the ability of a method to discriminate between them, hence its sensitivity, also changes ${ }^{4}$.

It is of especial interest, therefore, that a so-called 'negative' placebo effect is not necessarily an obstacle to the development of a useful and sensitive method of drug evaluation, for if the nature and the direction of placebo reaction, hence the base-line, can be identified, drug effect can be measured accurately enough; indeed, sometimes more accurately when they are opposed than when the effect of one obscures the effect of the other, as it may, when they develop simultaneously and in the same direction.

In the present instance, the dummy induced, not a typical placebo effect but, instead, a significant and useful stress, and the competitive mental work test merely provided an additional challenge, while the pharmacodynamic effect opposed both. It can be assumed that, regardless of how well they were re-assured that there was no hazard, the use of volunteer medical students ${ }^{5}$ who knew that they were participating in a drug investigation led to the unexpected and uniform placebo response. It is possible that, were the subjects entirely unaware of participation in an investigation and, more specifically, of drug testing, both placebo and pharmacodynamic actions might have been in the same, instead of opposite, directions. However this may be, this circumstance may nevertheless provide a wellcontrolled, sensitized setting for the evaluation of the small doses of tranquillizers so commonly used in general practice.

The importance of 'negative' placebo effects in simulating toxic effects of drugs has been stressed in the literature ${ }^{6}$. In the present case, such an effect has been measured and has been shown to be regular and predictable. Because of the several varieties of placebo reactions as well as their potential for interaction with pharmacodynamic effects of drugs $^{7}$, this dynamic quality, which is inseparable from the act of drug administration in man, must be taken into account, and controls must be used which identify the direction as well as the magnitude of placebo effects. For this it may be necessary to use the unblinded 'no-drug-at-all' control to complement the usual identical dummies in the double-blind setting.
This work was supported in part by the MeadJohnson Fund and in part by the Clinical Pharma. cology Fund of the Department of Pharmacology.

WALTER MODELL

MARgaret GarRETT*

Department of Pharmacology,

Cornell University Medical College, New York.

* Postdoctoral Fellow, U.S. Public Health Service Training Grant. ' Stroop, J. R., J. Exp. Psych., 18, 643 (1935).

${ }^{2}$ Gaddum, J. H., Proc. Roy. Soc. Med., 47, 195 (1954).

${ }^{3}$ Gold, H., Amer. J. Med., 17, 722 (1954).

${ }^{4}$ Modell, W., and Houde, R. W., J. Amer. Med. As8oc., 167, 2190 (1958).

s. Lasagna, Le, and von Telsinger, J. M., Science, 120, 359 (1954).

6 Wolf, S., and Pinsky, R. H., J. Amer. Med. Assoc., 155, 339 (1954). 'Wolff, H. G., and Goodell, H., Proc. Assoc. Res. Nerv. Neut. Dis., 23, 434 (i943).

\section{ANIMAL PHYSIOLOGY}

\section{Relation of Eye Dominance and Eye Closure}

IT is a well-known fact that while our vision is binocular, one eye (more often the right than the left) is in fact preferred or dominant in fixation and sighting. Much has been published on this subject, especially in its relation to handedness. We conjectured that if one eye is dominant in sighting, the other, that is, the non-dominant eye, would logically be expected to close more easily in order to enhance the use of the dominant eye, and as a corollary, the dominant eye would be more resistant to closure unilaterally because of the very nature of its dominance. In putting this theory to test, we used the students of our senior and junior anatomy classes (total 145, ranging from 18 to 24) as subjects. Eye dominance was first ascertained by three or more trials of the cone test in each case, and then the subjects were asked to close the right and left eyes singly and alternately for a number of times and to say which eye they found could close with less effort. This latter test was subjective; in some cases, however, we were able to see quite plainly for ourselves. The results of our investigation are given in Table 1.

\begin{tabular}{|c|c|c|}
\multicolumn{2}{c}{ Table 1 } \\
\hline No. of subjects & Dominant eye & Ease in closing \\
\hline 63 & $R$ & $L$ \\
31 & $L$ & $R$ \\
29 & $R$ & $R$ \\
16 & $L$ & $L$ \\
4 & $R$ & $N D$ \\
2 & $L$ & $N D$ \\
\hline
\end{tabular}

$L$, Left eye ; $R$, right eye; $N D$, no difference between the eyes.

The above results show that two-thirds of the subjects could close the non-dominant eye more easily than the dominant eye, while one-third the reverse. The value of chi-square obtained $\left(\chi^{2}=\right.$ 15.527 and $P=0.01$ ) shows a significant correlation between eye dominance and the relative ease of closure of the non-dominant eye. In addition, the results show that the differential function of the orbicularis oculi, innervated by the facial nerve, is a component in the dynamic complex of eye dominance.

S. T. ChaN

Department of Anatomy,

K. S. F. CHANG

University of Hong Kong,

Hong Kong. Nov. 28. 